\title{
Phytochemical Analysis of Anti-atherogenic Constituents of Xue-Fu-Zhu-Yu-Tang Using HPLC-DAD-ESI-MS
}

\author{
Li Liu, Yiyu CHENG, ${ }^{*}$ and Haijiang ZhANG \\ Pharmaceutical Informatics Institute, College of Pharmaceutical Sciences, Yuquan Campus, Zhejiang University; \\ Hangzhou 310027, China. Received June 7, 2004; accepted August 19, 2004
}

\begin{abstract}
Xue-Fu-Zhu-Yu-Tang (血府逐淤汤) is a famous traditional Chinese medicine (TCM) formula for treating cardiovascular disease and related ailments in China for centuries. To profile the phytochemical constituents of the formula, an HPLC-DAD-ESI-MS analytical method has been developed to separate and determinate the medium- or non-polar fraction of the decoction, which has been demonstrated potency to lower the serum total triglyceride concentration, strongly decrease the $\mathrm{TXA}_{2} / \mathrm{PGI}_{2}$ ratio and attenuate production of proinflammatory cytokines in high cholesterol-fed rats. By comparing their retention time, UV and MS data with those obtained from the authentic compounds, ferulic acid (1), naringin (2), neohesperindin (3), naringenin (8), marmin (13), senkyunolide A (14), dehydrosafynol (16), safynol (17) and $Z$-ligustlide (18) are unequivocally determined. Moreover, additional thirteen compounds are tentatively identified as senkyunolide $I$ (4), senkyunolide $H$ (5), poncirin (7), benzoylpaeoniflorin (10), $(Z)-6,7$-epoxyligustilide (11), senkyunolide $G$ (12), 2-methoxy-safynol (15), cnidilide (19), tangeritin (20), saikosaponin $b_{2}(21), 2^{\prime}$ - $O$-acetylsaikosaponin $b_{2}(22)$, saikosaponin $b_{1}(23)$ and auraptene (24), according to the comparison of their UV and MS data with the published data. The present study provides an approach to rapidly characterize bioactive constituents in TCM formulae.
\end{abstract}

Key words HPLC-DAD-MS; traditional Chinese medicine (TCM); atherosclerosis; herbal formula

In China, traditional Chinese medicines (TCMs) have already been used for centuries in the treatment of a wide variety of ailments successfully and have attracted more and more attention from industry and academia. ${ }^{1-3)}$ However, their remedial mechanisms are still not well understood. So far, it is widely accepted that multiple ingredients are responsible for therapeutic effects of TCMs. Thus, it is necessary to clarify and analyze the bioactive constituents in TCMs to ensure the reliability in clinical application, to better understand the pharmacological basis and moreover, to enhance products quality control.

However, research on TCMs presents significant challenges, especially for herbal formulae, due to the complexity of chemical composition. In recent years, the advent of HPLC coupled mass spectrometer has had tremendous impact upon the profile analysis of natural products and drug discovery. ${ }^{4-7)}$ HPLC-DAD-ESI-MS has been successfully applied to the determination of compounds present in material from a variety of natural product sources. ${ }^{8-11)}$ A significant number of reports have been published on Chinese medicinal herbs using this hyphenated technique, ${ }^{12}$ although only few on TCM formulae.

Xue-Fu-Zhu-Yu-Tang is a famous Chinese TCM formula for treating cardiovascular disease with a history of several centuries. The formula mainly consists of six plant materials: Paeonia lactiflora, Ligusticum chuanxiong, Citrus aurantium, Carthamus tinctorius, Prunus persicae and Bupleurum falcatum. Satisfactory therapeutic effect has been achieved especially for atherosclerosis and hyperlipidemia. ${ }^{13)}$ But the bioactive principles of this formula are still not clarified as yet.

In this paper, we describe a study on phytochemical constituents of bioactive fraction of Xue-Fu-Zhu-Yu-Tang. Considering the criteria for drug-like compounds, ${ }^{14}$ ) especially hydrophobic points and molecular weight range, we suppose that medium- or non-polar constituents of the formula might have significant bioactivity. It was demonstrated using a high cholesterol-fed animal model. And then, using an HPLCDAD-ESI-MS method, 22 major constituents were identified in the fraction, based on the comparison of their UV and MS data with those of authentic compounds and published data. These results may help to gained better understanding of therapeutic basis of Xue-Fu-Zhu-Yu-Tang and establish a better quality control method for the old-age TCM formula.

\section{Experimental}

Chemicals and Reagents HPLC grade methanol (E. Merck, Darmstadt, Germany) was used for HPLC analysis. Reagent grade ethanol (Hangzhou Reagent Company, Hangzhou, China) was used for extraction and separation. The D101 macro-porous resin (Tianjing Resin Factory, Tianjing, China) was used for the chromatographic separation of the formulation and plant materials.

Materials and Sample Preparation The plant materials: Paeonia lactiflora, Ligusticum chuanxiong, Citrus aurantium, Carthamus tinctorius, Prunus persicae and Bupleurum falcatum, were provided and identified by China Academy of Traditional Chinese Medicine (Beijing, China). The six plant materials (the total weight of $20 \mathrm{~kg}$ ) were mixed according to traditional formula, and then decocted for 3 times (for $2 \mathrm{~h}$ each time). After filtration and concentration, aqueous extract (AE) was evaporated to dryness. The yield of the preparation was $16.5 \% .1 \mathrm{~kg}$ dried extract was re-dissolved in water and separated on D101 porous resin columns. A step gradient of (1) $\mathrm{H}_{2} \mathrm{O}$, (2) $90 \% \mathrm{H}_{2} \mathrm{O}, 10 \% \mathrm{EtOH}$, (3) $70 \% \mathrm{H}_{2} \mathrm{O}, 30 \% \mathrm{EtOH}$, (4) $50 \% \mathrm{H}_{2} \mathrm{O}$, $50 \% \mathrm{EtOH},(5) 30 \% \mathrm{H}_{2} \mathrm{O}, 70 \% \mathrm{EtOH}$, (6) $5 \% \mathrm{H}_{2} \mathrm{O}, 95 \% \mathrm{EtOH}$ was applied as mobile phase. The last fraction was collected as the medium-polar and non-polar fraction (MNPF). The yield of MNPF was about $1 \%$ of the aqueous extract. The corresponding extracts of individual plant material (each weight of $100 \mathrm{~g}$ ) were also prepared with the same procedure.

HPLC-DAD-ESI-MS Condition The analysis was performed using an Agilent 1100 series HPLC-DAD-MS system (Aglient Technologies, Willington, U.S.A.). A Zorbax StableBond C18 column $(4.6 \mathrm{~mm} \times 250 \mathrm{~mm}$, $5 \mu \mathrm{m}$, Aglient) with an C18 guard column (Hanbon Science \& Technology Co., Ltd, Jiangsu, China) was utilized. Samples including MNPF from formula and individual plant material were dissolved in $\mathrm{MeOH}$ as $5 \mathrm{mg} / \mathrm{ml}$ solutions and centrifuged at $12000 \mathrm{rpm}$ for $15 \mathrm{~min}$ to remove particles before injection. The mobile phase consisted of (A) $0.8 \%$ aqueous acetic acid and (B) methanol using a linear gradient (from $40 \% \mathrm{~B}$ to $90 \% \mathrm{~B}$ in $100 \mathrm{~min}$ ). The flow rate was $0.5 \mathrm{ml} / \mathrm{min}$. The temperature of column was $30^{\circ} \mathrm{C}$. The UV spectrum was recorded from 190 to $400 \mathrm{~nm}$. Two separate injections for each sample were performed in positive ion mode and negative ion mode respectively with electrospary ionization (ESI). The full scan mass spectrum 
Table 1. Hypolipidemia Effect of Aqueous Extract and MNPF in High Cholesterol-Fed Rats

\begin{tabular}{lllll}
\hline \hline Group & \multicolumn{1}{c}{ TC } & \multicolumn{1}{c}{ TG } & HDL-C & LDL-C \\
\hline Control & $1.57 \pm 0.23^{* *}$ & $0.41 \pm 0.14$ & $1.02 \pm 0.19$ & $0.48 \pm 0.25^{* *}$ \\
Hyper & $5.40 \pm 2.64$ & $0.50 \pm 0.11$ & $1.21 \pm 0.41$ & $1.60 \pm 0.38^{* *}$ \\
AE & $3.00 \pm 1.80$ & $0.34 \pm 0.14^{*}$ & $1.08 \pm 0.37$ & $4.08 \pm 2.44$ \\
MNPF & $3.71 \pm 2.17$ & $0.31 \pm 0.08^{* *}$ & $1.24 \pm 0.43$ & $1.73 \pm 1.64^{*}$ \\
\hline
\end{tabular}

Values are expressed as mean \pm S.D. for ten animals in each group. The values are expressed as mmol/1 serum. $*$ Different $v s$. hyper group $(p<0.05) ; * *$ different $v s$. hyper group $(p<0.01)$.

Table 2. Anti-coagulation Effect and Inhibition of Inflammatory Cytokines Release of Aqueous Extract and MNPF in High Cholesterol-Fed Rats

\begin{tabular}{|c|c|c|c|c|c|}
\hline Group & 6-keto-PGF ${ }_{1 \mathrm{a}}$ & $\mathrm{TXB}_{2}$ & TXB2/6-keto-PGF 1 a & IL-6 & IL-8 \\
\hline Control & $626.45 \pm 166.20 * *$ & $445.78 \pm 131.24$ & $0.83 \pm 0.39 * *$ & $0.102 \pm 0.059^{*}$ & $0.245 \pm 0.075$ \\
\hline Hyper & $365.94 \pm 80.95$ & $589.42 \pm 172.01$ & $1.68 \pm 0.55$ & $0.183 \pm 0.052$ & $0.289 \pm 0.044$ \\
\hline $\mathrm{AE}$ & $464.86 \pm 187.78$ & $487.11 \pm 109.74$ & $1.13 \pm 0.50 *$ & $0.177 \pm 0.103$ & $0.243 \pm 0.012$ \\
\hline MNPF & $536.48 \pm 193.01 *$ & $442.45 \pm 200.96$ & $0.79 \pm 0.20 * *$ & $0.141 \pm 0.056$ & $0.130 \pm 0.066^{* *}$ \\
\hline
\end{tabular}

Values are expressed as mean \pm S.D. for ten animals in each group. Lever of $\mathrm{TXB}_{2}$ and 6-keto-PGF ${ }_{1 \mathrm{a}}$ are expressed as $\mathrm{pg} / \mathrm{ml}$ serum. Lever of IL-8 and IL-6 are expressed as $\mathrm{ng} / \mathrm{ml}$ serum. $*$ Different $v s$. hyper group $(p<0.05) ; * *$ different $v s$. hyper group $(p<0.01)$.

was recorded over the range of $m / z 100-1200$. Temperature of drying gas was $350^{\circ} \mathrm{C}$ with gas flow rate of $10.01 / \mathrm{min}$ and a nebulising pressure of $45 \mathrm{psi}$. The value of fragmentor voltage was set at 120 .

Reference Compounds Ferulic acid (1), naringin (2), neohesperindin (3), naringenin (8) were purchased from the National Institute for the Control of Pharmaceutical and Biological Products (Beijing, China). Senkyunolide A (14) and Z-ligustilide (18) were isolated from Ligusticum chuanxiong according to Kobayashi's description. ${ }^{15)}$ Marmin (13) was isolated from Citrus aurantium by the following procedures. The plant material $(100 \mathrm{~g})$ was refluxed with $70 \% \mathrm{EtOH}(500 \mathrm{ml})$ for 3 times. The free solvent extract was subjected to preparative TLC and semi-preparative RP-HPLC for separation and purification. The developing solvents for preparative TLC were $\mathrm{CHCl}_{3}$ $\mathrm{MeOH}(10: 1, \mathrm{v} / \mathrm{v})$. The mobile phase for semi-preparative HPLC was $60 \%$ methanol in water. Dehydrosafynol (16) and safynol (17) were isolated from Carthamus tinctorius according to Redl's discription. ${ }^{16)}$ The purity of each isolated compound was determined by HPLC analysis. And their identities were confirmed by the comparison of their ${ }^{1} \mathrm{H}-\mathrm{NMR}$ spectrum with the published data. ${ }^{15-23)}$

Animials and Treatment Male Wistar rats $(n=40)$ weighing 160 $190 \mathrm{~g}$, obtained from the Chinese Academy of Sciences (Beijing, China) were randomly divided into two groups and housed in cages random, with water and food freely available. The first group (control group, $n=10$ ) received a control diet and another group (hyperlipidemia model group, $n=30$ ) was fed on a diet containing $4 \%$ cholesterol, $10 \%$ lard, $0.2 \%$ methylthiouracil and $0.8 \%$ cholic acid throughout the overall experimental period ( $21 \mathrm{~d}$ ). After $7 \mathrm{~d}$, the first hyperlipidemia group rats (AE group, $n=10)$ were given AE orally ( $8 \mathrm{~g} / \mathrm{kg}$ per day), the second hyperlipidemia group rats (MNPF group, $n=10)$ were given MNPF orally $(0.2 \mathrm{~g} / \mathrm{kg}$ per day), the third hyperlipidemia group rats (hyper group, $n=10$ ) were given physiological saline, for $14 \mathrm{~d}$.

Measurement of Biochemical Parameters At the end of the experimental trial the rats were fasted for $12 \mathrm{~h}$. The blood samples were withdrawn from the abdominal aorta and then transferred directly into centrifuge tubes and allowed to clot at room temperature and centrifuged for $15 \mathrm{~min}$ at $3000 \mathrm{rpm}$. The supernatant clear serum concentration of total cholesterol (TC) was measured by CEH-COD-POD assay kit (Ningbo Asia-pacific Biotehnology Ltd., Ningbo, China), triglyceride (TG) was measured by GKGPO-POD assay kit (Ningbo Asia-pacific Biotehnology Ltd., Ningbo, China). HDL-C was measured by PEG-modified enzyme HDL-C assay and LDL-C was measured by solubilization LDL-C assay (Roche Diagnostics, Switzerland). $\mathrm{TXB}_{2}$ and 6-keto-PGF ${ }_{1 \mathrm{a}}$ were measured by EIA (Cayman Chemical Company, Ann Arbor, U.S.A.). IL-8 and IL-6 were measured by ELISA (Pierce Biotechnology Inc., Rockford, U.S.A.).

Statistical Analyses The results are expressed as mean \pm S.D. and they were evaluated statistically using the one-way analysis of variance (ANOVA) followed by the Dunnett $t$-test. In all cases, $p<0.05$ was regarded as significance and $p<0.01$ was regarded as very significance.

\section{Results and Discussion}

Bioassays for Anti-atherogenic Fraction A commonly used high cholesterol-fed rat mode ${ }^{24)}$ was applied in the investigation. Three sectors of biochemical parameters were selected to evaluate anti-atherogenic effect of samples: serum lipid lever ${ }^{25)}$ blood coagulation potential ${ }^{26)}$ and proinflammatory cytokine concentration, ${ }^{27)}$ which were well-known factors in pathogenesis of atherosclerosis. The results presented in Tables 1 and 2 indicated that AE of the formula significantly lowered triglyceride concentration and decreased $\mathrm{TXA}_{2} / \mathrm{PGI}_{2}$ ratio (measured by assays for $\mathrm{TXB}_{2}$ and 6-keto$\mathrm{PGF}_{1 \mathrm{a}}$ ). And MNPF of the formula exhibited comparable effect on serum lipid lever. Furthermore, it showed stronger potency to increase the $\mathrm{PGI}_{2}$ secretion and significantly inhibitory effect on IL-8 production. Considering that the treatment dose in MNPF group was $2.5 \%$ of that of AE group, we suggested that MNPF was the anti-atherogenic principle of the formula.

HPLC-DAD-ESI-MS Analysis of MNPF An HPLCDAD-ESI-MS method was developed to determine the phytochemical constituents of the MNPF. As shown in Fig. 1A (overlapping chromatogram of 254, 280 and $320 \mathrm{~nm}$ ), 24 major peaks were separated under the HPLC condition with UV detection. The chromatography of MS total ion current (TIC) in positive mode and negative mode was shown in Fig. $1 \mathrm{~B}$ and Fig. 1C separately. The retention time, $\mathrm{m} / \mathrm{z}$ value and UV maximum adsorption wavelength of each peak was summarized in Table 3.

Phenolic and Flavonoid Compounds Ferulic acid, naringin, neohesperindin and naringenin were unequivocally determined by comparison with authentic compounds. Peak 7 exhibited UV spectrum with maximum absorption at 226, 284 and $326 \mathrm{~nm}$, indicating that the compound was a saturated flavanone (Fig. 2A). According to additional information about its plant origin and MS data, the peak was assigned as poncirin. ${ }^{28)}$ Naringin, neohesperindin and poncirin exhibited not only intense quasi-molecular ions $[\mathrm{M}+\mathrm{H}]^{+}$and sodiated adduct ions $[\mathrm{M}+\mathrm{Na}]^{+}$, but also significant aglycone molecular ion (Figs. $3 \mathrm{~A}-\mathrm{C}$ ) in positive mode. Peak 20 exhibited intense $[\mathrm{M}+\mathrm{H}]^{+}$with adduct ion $[\mathrm{M}+\mathrm{Na}]^{+}$and dimmer ion $[2 \mathrm{M}+\mathrm{Na}]^{+}$in positive mode, but weak response in 
A

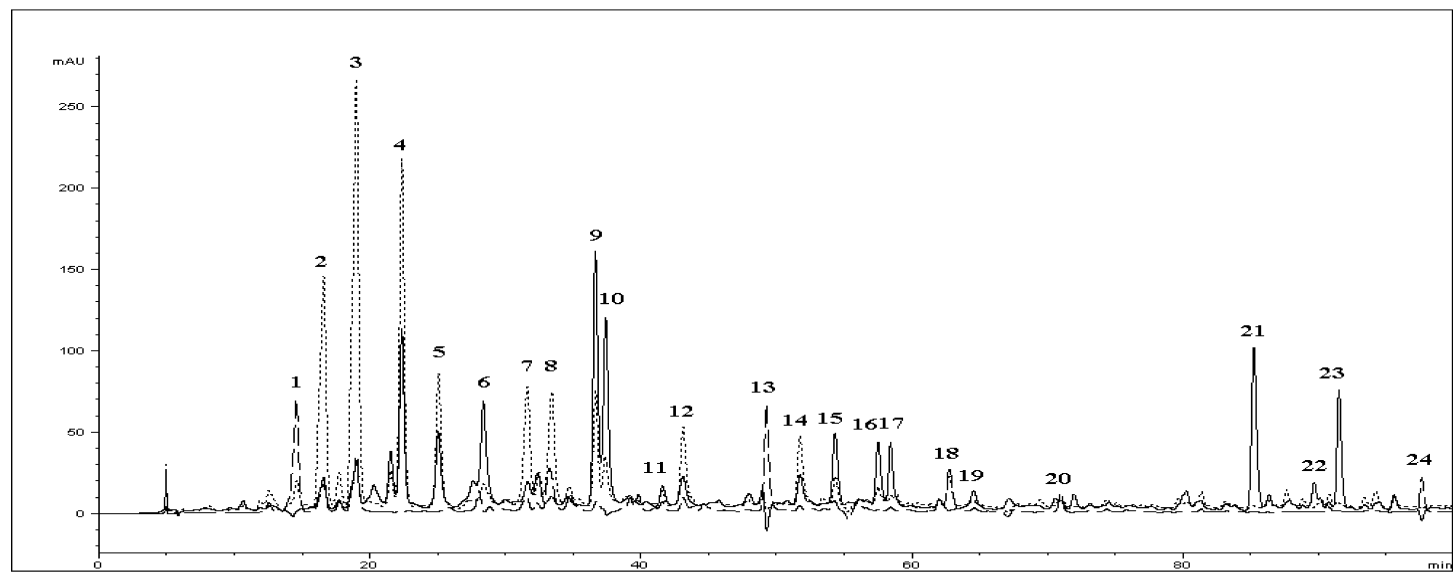

B

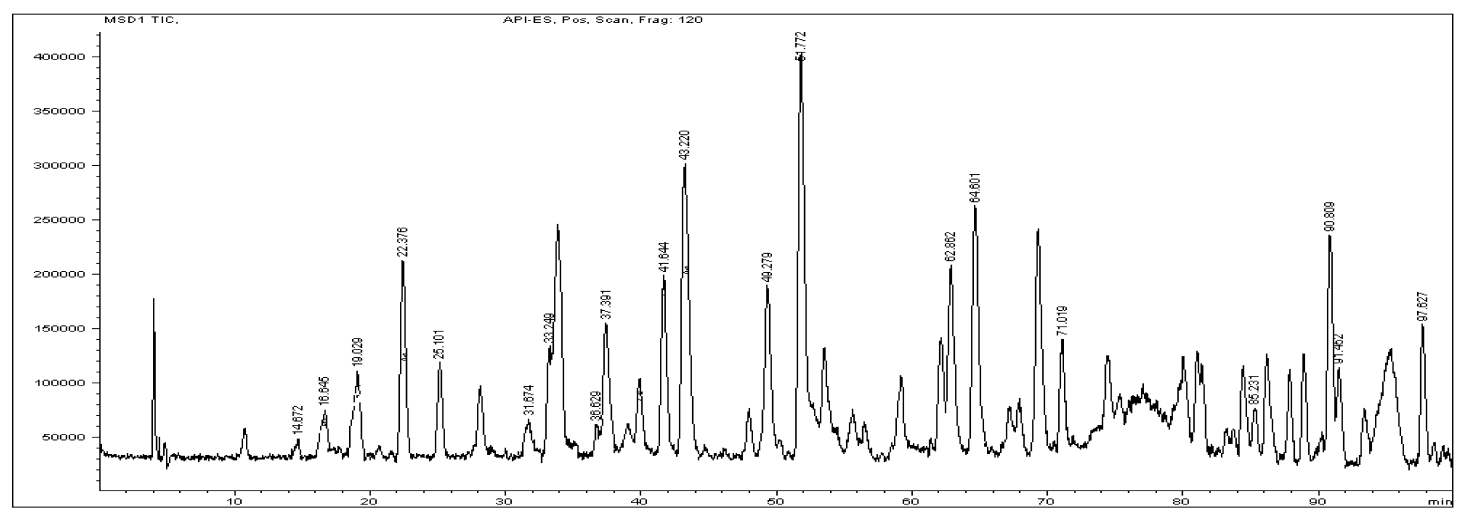

C

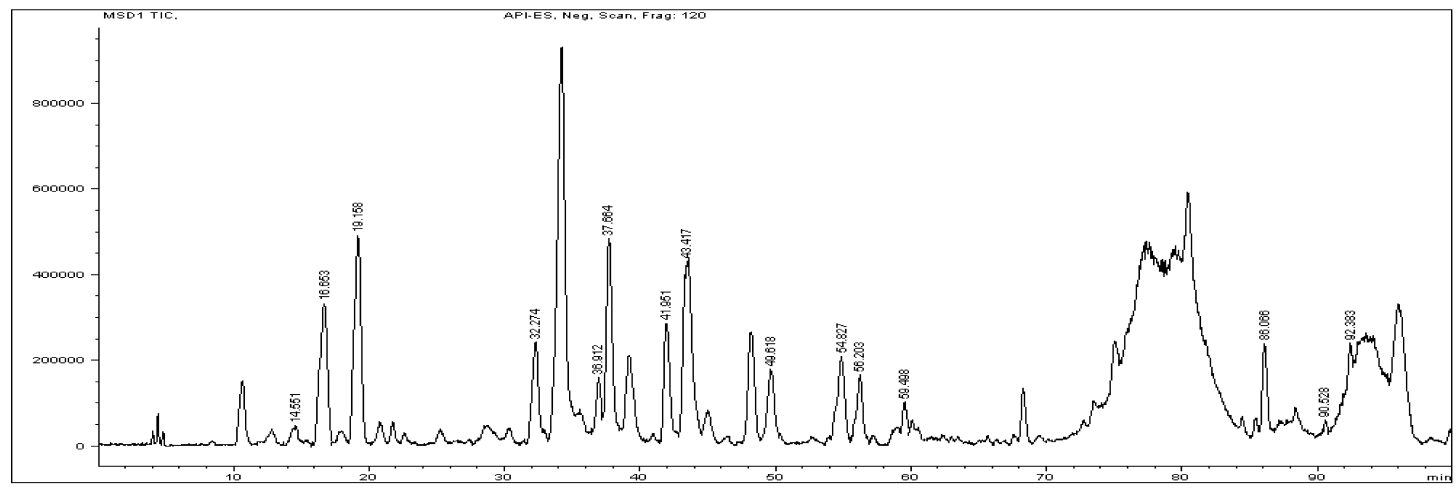

Fig. 1. Chromatogram of MNPF of Xue-Fu-Zhu-Yu-Tang

(A) Overlapping chromatogram of $254 \mathrm{~nm}$ (solid line), $280 \mathrm{~nm}$ (dot line) and $320 \mathrm{~nm}$ (dash line) of MNPF. 1: ferulic acid, 2: naringin, 3: neohesperindin, 4 and 5: senkyunolide I or senkyunolide H, 6: unknow, 7: poncirin, 8: naringenin, 9: unknow, 10: benzoylpaeoniflorin, 11: (Z)-6,7-epoxyligustilide, 12: senkyunolide G, 13: marmin, 14: senkyunolide A, 15: 2-methoxy-safynol, 16: dehydrosafynol, 17: safynol, 18: $Z$-ligustlide, 19: cnidilide, 20: tangeritin, 21 and 23: saikosaponin $b_{1}$ or saikosaponin $b_{2}$, 22: 2 '- $O$-acetylsaikosaponin $\mathrm{b}_{2}$, 24: auraptene. (B) TIC chromatogram in positive mode of MNPF (retention time of the identified peak was indicated). (C) TIC chromatogram in negative mode of MNPF (retention time of the identified peak was indicated).

negative mode. It was tentatively inferred that the peak was tangeritin based on its molecular mass and UV spectrum, which agreed with the published data. ${ }^{9}$

Phthalides Seven phthalides found in MNPF of Xue-FuZhu-Yu-Tang were assigned to Ligusticum chuanxiong. Except senkyunolide $\mathrm{A}$ and $Z$-ligustlide, the other five peaks were tentatively determined based on comparison their UV and MS data with published data. ${ }^{15,17,29-31)}$ Peak 4 and peak
5 exhibited a dehydrating ion $\left[\mathrm{M}+\mathrm{H}-\mathrm{H}_{2} \mathrm{O}\right]^{-}$indicated the structure dihydroxylated at C-6 and C-7 (Fig. 3D). UV and MS data of the two peaks were in good agreement with the published data for senkyunolide I and senkyunolide $\mathrm{H}^{10)}$ Since the UV and MS data could not provide stereochemistry information, the two compounds were not differentiated. (Z)6,7-Epoxyligustilide and senkyunolide $\mathrm{G}$ showed protonated molecular ion $[\mathrm{M}+\mathrm{H}]^{+}$, sodiated monomer $[\mathrm{M}+\mathrm{Na}]^{+}$and 
Table 3. HPLC-DAD-ESI-MS Identification

\begin{tabular}{|c|c|c|c|c|c|c|c|c|}
\hline Peak & $\begin{array}{c}t_{\mathrm{R}}^{a)} \\
(\mathrm{min})\end{array}$ & {$[\mathrm{M}+\mathrm{H}]^{+}$} & $\begin{array}{l}\text { Other ions } \\
\left(\mathrm{ESI}^{+}\right)\end{array}$ & {$[\mathrm{M}-1]^{-}$} & $\begin{array}{l}\text { Other ions } \\
\left(\mathrm{ESI}^{-}\right)\end{array}$ & $\lambda_{\max }(\mathrm{nm})$ & $\begin{array}{c}\text { Plant } \\
\text { material }\end{array}$ & Identify \\
\hline 1 & 14.53 & 197 & 217 & 193 & & 236,323 & $\mathrm{LC}$ & Ferulic $\operatorname{acid}^{b)}$ \\
\hline 2 & 16.53 & 581 & 603 & 579 & 615 & 283,328 & CA & Naringin $\left.^{b}\right)$ \\
\hline 3 & 18.95 & 611 & 633 & 609 & 645 & 284,328 & $\mathrm{CA}$ & Neohesperindin ${ }^{b)}$ \\
\hline 4 & 22.31 & 225 & $207,247,471$ & 223 & & 275 & $\mathrm{LC}$ & Senkyunolide $\mathrm{I}^{c)}$ \\
\hline 5 & 25.02 & 225 & $207,247,471$ & 223 & 259,283 & 276 & $\mathrm{LC}$ & Senkyunolide $\mathrm{H}^{c)}$ \\
\hline 6 & 28.33 & 317 & & 315 & & 252,372 & $\mathrm{~N}$ & Unknown \\
\hline 7 & 31.57 & 595 & 617 & 593 & 629,653 & $226,284,326$ & CA & Poncirin \\
\hline 8 & 33.37 & 273 & & 271 & & 286,328 & $\mathrm{CA}$ & Naringenin $^{b)}$ \\
\hline 9 & 36.60 & 331 & 353,683 & 329 & & 250 & PL & Unknown \\
\hline 10 & 37.36 & 585 & 607 & 583 & 643,1167 & 234 & PL & Benzoylpaeoniflorin \\
\hline 11 & 41.93 & 207 & 229,435 & 205 & & 276 & $\mathrm{LC}$ & (Z)-6,7-Epoxyligustilide \\
\hline 12 & 43.07 & 209 & 231,439 & 207 & & 284 & $\mathrm{LC}$ & Senkyunolide G \\
\hline 13 & 49.21 & 333 & 315,355 & & 367,391 & 324 & $\mathrm{CA}$ & Marmin $^{b)}$ \\
\hline 14 & 51.69 & 193 & 215,407 & & & 281 & $\mathrm{LC}$ & Senkyunolide $\mathrm{A}^{b)}$ \\
\hline 15 & 54.29 & & 237 & & 249,273 & $258,268,324,348$ & $\mathrm{CT}$ & 2-Methoxy-safynol \\
\hline 16 & 57.46 & & 181,221 & 197 & 233,257 & $240,256,270,304$ & $\mathrm{CT}$ & Dehydrosafynol $^{b)}$ \\
\hline 17 & 58.38 & & 223 & 199 & 235,259 & $246,254,268,308$ & $\mathrm{CT}$ & Safynol $^{b)}$ \\
\hline 18 & 62.72 & 191 & 213,403 & & & 280,328 & $\mathrm{CT}$ & $Z$-Ligustlide ${ }^{b)}$ \\
\hline 19 & 64.52 & 195 & 217,411 & & & 234,315 & $\mathrm{LC}$ & Cnidilide \\
\hline 20 & 71.92 & 373 & 395,767 & & & 272,323 & $\mathrm{CA}$ & Tangeritin \\
\hline 21 & 85.19 & & 763,803 & 779 & 815 & $244,252,262$ & $\mathrm{BF}$ & Saikosaponin $\mathrm{b}_{2}{ }^{c}$ \\
\hline 22 & 89.64 & & 845 & 821 & & $244,252,262$ & $\mathrm{BF}$ & $2^{\prime}-O$-Acetylsaikosaponin $b_{2}$ \\
\hline 23 & 91.47 & & 763,803 & 779 & & $244,250,260$ & $\mathrm{BF}$ & Saikosaponin $\mathrm{b}_{1}{ }^{c}$ \\
\hline 24 & 97.57 & 299 & 321,619 & 297 & & 325 & $\mathrm{CA}$ & Auraptene \\
\hline
\end{tabular}

a) Retention time of peaks in Fig. 1A. b) Unequivocally determined by comparison with authentic compounds. c) Not able to differentiate absolute configuration. The abbreviation for plant material: PL vs. Paeonia lactiflora; LC vs. Ligusticum chuanxiong; CT vs. Carthamus tinctorius; CA vs. Citrus aurantium; SP vs. Prunus persicae; BF vs. Bupleurum falcatum; $\mathrm{N}$ means not found in any plant material.
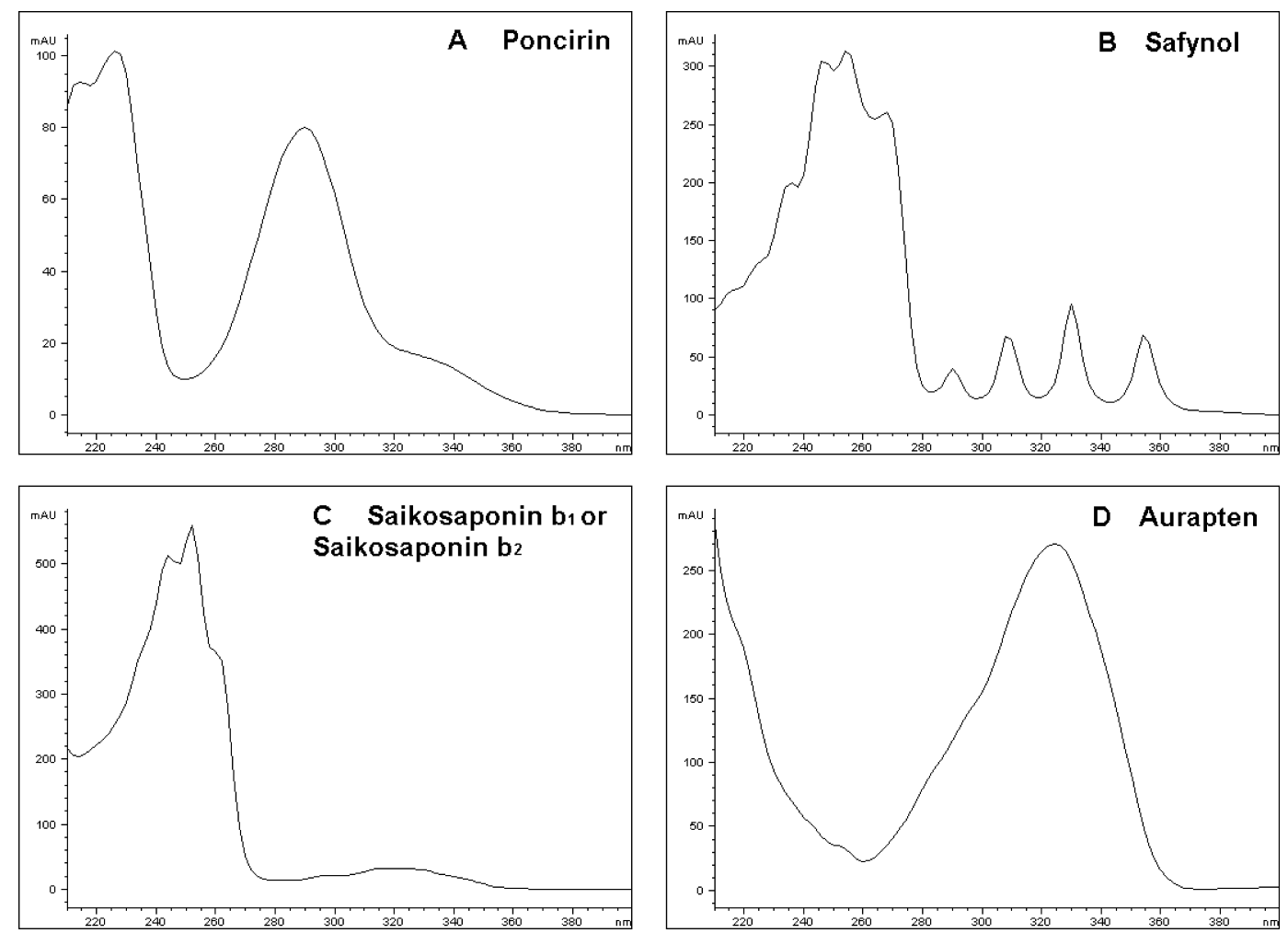

Fig. 2. Typical UV Spectrum of Saturated Flavanone, Polyacetylene, Saponin and Coumarins

dimer ions $[2 \mathrm{M}+\mathrm{Na}]^{+}$in positive mode, and intense quasimolecular ions $[\mathrm{M}-\mathrm{H}]^{-}$(in negative mode. But for senkyunolide A, Z-ligustlide and cnidilide, the responses in positive mode were much stronger than those in negative mode.

Monoterpene Glycoside One monoterpene was found in MNPF of the formula, namely peak 10, which was from Paeonia lactiflora. The peak exhibited very intense quasimolecular ions $[\mathrm{M}-\mathrm{H}]^{-}$, adduct ion $[\mathrm{M}+\mathrm{AcO}]^{-}$and dimmer ion $[2 \mathrm{M}-\mathrm{H}]^{-}$in negative mode. The molecular mass was inferred to be 584, which was confirmed by MS data ob- 
A Naringin

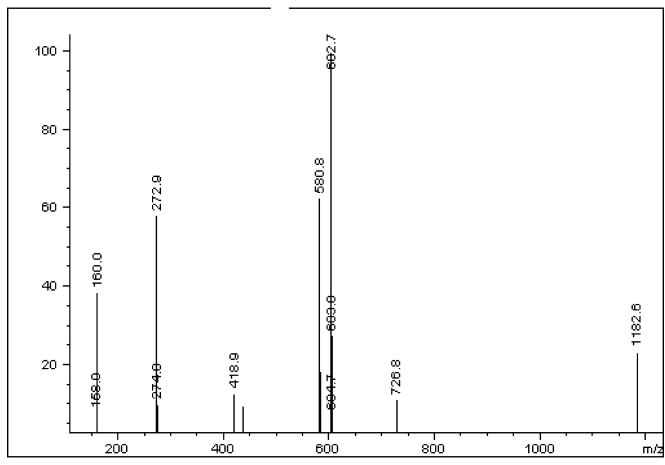

\section{Poncinin}

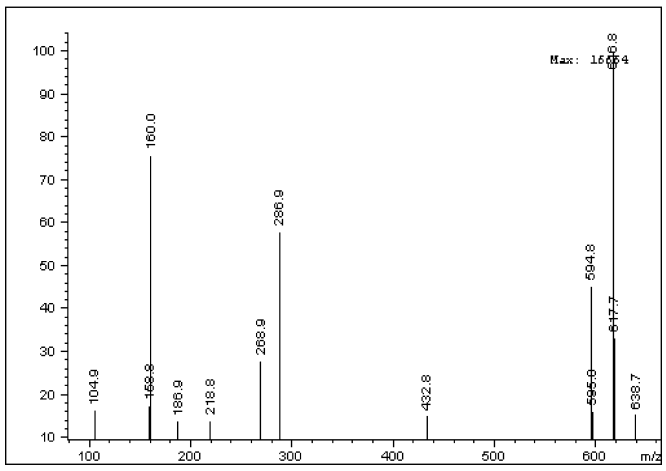

\section{E Saikosaponin $b_{1}$ or Saikosaponin $b_{2}$}

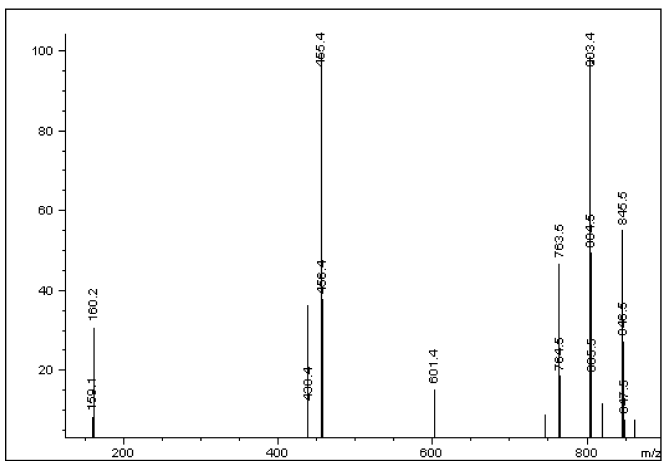

Fig. 3. MS Spectrum of Several Identified Compounds in Positive Mode

tained in positive mode. The typical UV spectrum with maximum absorption at $236 \mathrm{~nm}$ corresponded to the structure of benzoylpaeoniflorin. ${ }^{32)}$

Polyacetylenes Typical characters of polyacetylene UV spectrum were observed in peak 15, 16 and 17 (Fig. 2B). According to comparison with individual plant material, the three peaks were assigned to Carthamus tinctorius. Peak 16 and 17 were unequivocally determined to be dehydrosafynol and safynol respectively by comparison with authentic compounds. From the presence of $[\mathrm{M}+\mathrm{AcO}]^{-}$in negative mode and $[\mathrm{M}+\mathrm{Na}]^{+}$in positive mode, the molecular mass of peak 15 was inferred to be 214 . The UV and MS data corresponded to 2-methoxylsafynol, which was found in Centaurea ruthenica previously. ${ }^{33}$ The polyacetylenes gave significant adduct ion $[\mathrm{M}+\mathrm{AcO}]^{-}$and weak quasi-molecular ions $[\mathrm{M}-\mathrm{H}]^{-}$in negative mode, whereas the responses in positive mode were very weak.

Saponins According to comparison of retention time,

\section{B Neohesperindin}

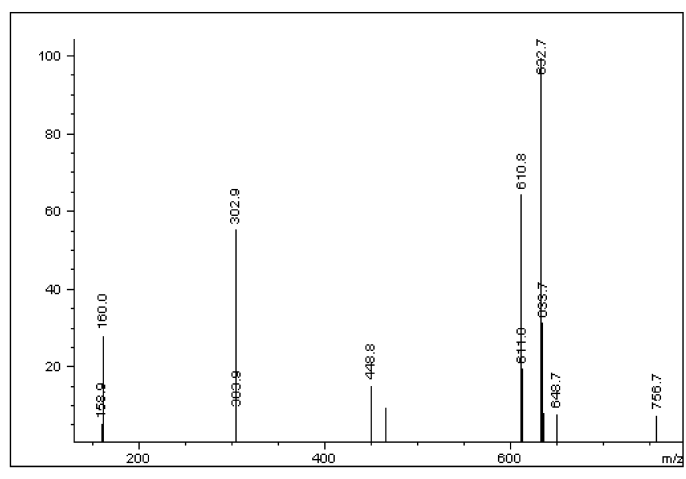

\section{Senkyunolide I or Senkyunolide H}

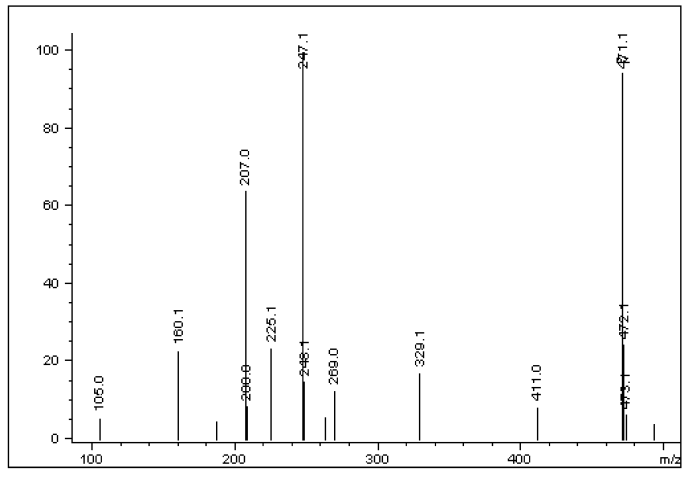

\section{F 2'-O-acetylsaikosaponin $b_{2}$}

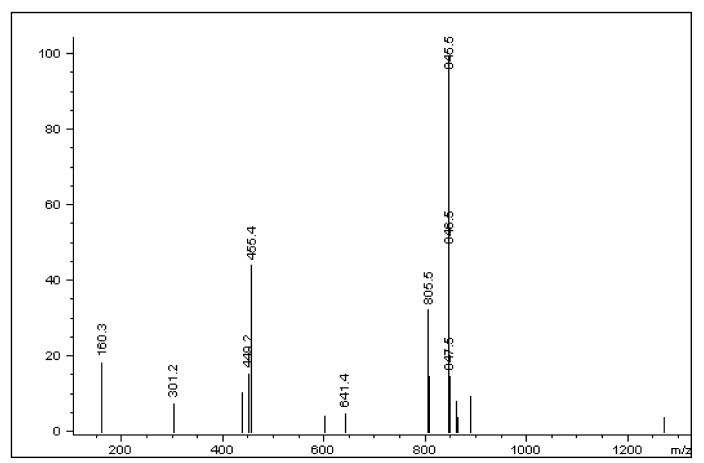

UV and MS data with those of individual plant material, peak 21, 22 and 23 were assigned to Bupleurum falcatum. The UV spectrum of the three peaks with absorption maximum at 244, 252 (250) and 262 (260) nm (Fig. 2C), indicated the existence of conjugated heteroannular diene. Peak 21 and 23 exhibited intense $[\mathrm{M}+\mathrm{Na}]^{+}$and significant $[\mathrm{M}+\mathrm{H}-$ $\left.\mathrm{H}_{2} \mathrm{O}\right]^{+}$together with fragments of $\left[\mathrm{M}+\mathrm{H}-\mathrm{H}_{2} \mathrm{O}-\mathrm{Glc}\right]^{+}$at $\mathrm{m} / \mathrm{z}$ of 601 and $\left[\mathrm{M}+\mathrm{H}-\mathrm{H}_{2} \mathrm{O}-\mathrm{Glc}-\mathrm{Fuc}\right]^{+}$at $\mathrm{m} / \mathrm{z}$ of 455 in positive mode (Fig. 3E). These data were in agreement with those of saikosaponin $b_{1}$ and saikosaponin $b_{2}$, which had been found in Bupleurum falcatum. ${ }^{34)}$ But we could not differentiate the two compounds due to insufficient stereochemistry information provided. Peak 22 exhibited $[\mathrm{M}+\mathrm{Na}]^{+}$at $m / z$ of $845,\left[\mathrm{M}+\mathrm{H}-\mathrm{H}_{2} \mathrm{O}\right]^{+}$at $m / z$ of 805 , and a dehydrating aglycone ions at $\mathrm{m} / \mathrm{z}$ of 455 (Fig. $3 \mathrm{~F}$ ), which corresponded to the structure of $2^{\prime}$ - $O$-acetylsaikosaponin $\mathrm{b}_{2}{ }^{35)}$

Coumarins Peak 13 and 24 were assigned to Citrus aurantium. The two peaks exhibited very similar UV spectrum 

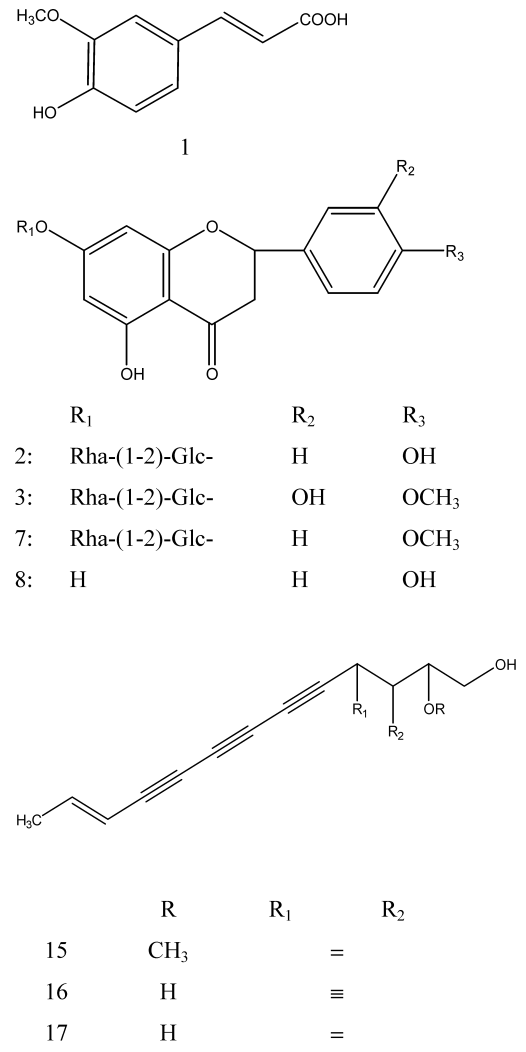

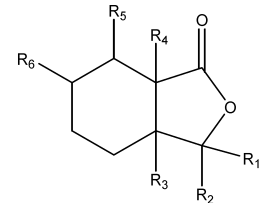

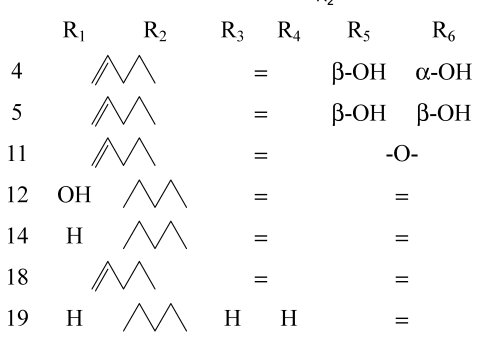

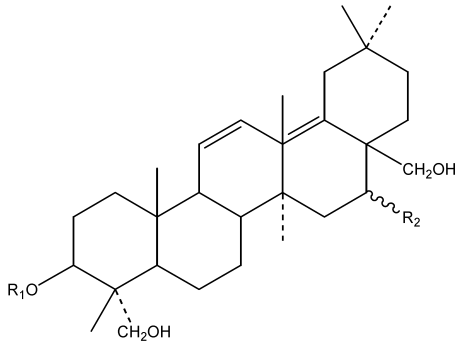

$$
\begin{array}{lll} 
& \mathrm{R}_{1} & \mathrm{R}_{2} \\
21 / 23 & \text { Glc-(1-3)-Fuc- } & \alpha-\mathrm{OH} \\
22 & \text { O-acetyl-Glc-(1-3)-Fuc- } & \alpha-\mathrm{OH} \\
23 / 21 & \text { Glc-(1-3)-Fuc- } & \beta-\mathrm{OH}
\end{array}
$$

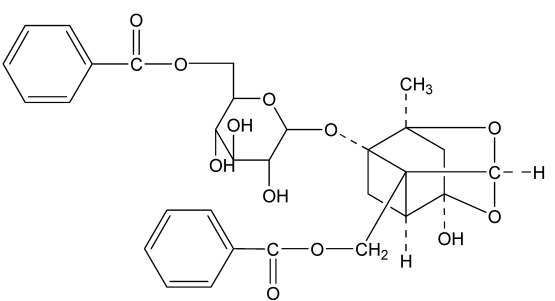

10<smiles>[2H]C(CC/C(C)=C/COc1ccc2ccc(=O)oc2c1)C(C)(C)C</smiles><smiles></smiles><smiles>COc1ccc(-c2cc(=O)c3c(OC)c(OC)c(OC)c(OC)c3o2)cc1</smiles>

20

Fig. 4. Structure of Compounds Identified

Ferulic acid (1), naringin (2), neohesperindin (3), senkyunolide I (4), senkyunolide H (5), Poncirin (7), naringenin (8), mudanoside (9), benzoylpaeoniflorin (10), (Z)-6,7-epoxyligustilide (11), senkyunolide G (12), marmin (13), senkyunolide A (14), 2-methoxy-safynol (15), dehydrosafynol (16), safynol (17), Z-ligustlide (18), cnidilide (19), tangeritin (20), saikosaponin $\mathrm{b}_{2}(\mathbf{2 1}), 2^{\prime}$ - $O$-acetylsaikosaponin $\mathrm{b}_{2}(\mathbf{2 2})$, saikosaponin $\mathrm{b}_{1}(\mathbf{2 3})$, auraptene (24).

with absorption maximum at $324 \mathrm{~nm}$ (Fig. 2D), suggested the presence of coumarin moiety. Peak 13 was unequivocally determined as marmin by comparing with authentic compounds. Peak 24 showed significant response in both positive mode and negative mode. The molecular mass was inferred to be 298. We tentatively assagened it as aurapten, considering that its UV and MS data was in agreement with published literature. ${ }^{36)}$

As a result, 22 compounds showed in Fig. 4 were determined and assigned to plant materials: the flavonoids and coumarins were mainly from Citrus aurantium, phthalides were mainly from Ligusticum chuanxiong, polyacetylenes were from Carthamus tinctorius, saponins were from $\mathrm{Bu}$ pleurum falcatum. Moreover, a monoterpene glycoside was assigned to Paeonia lactiflora. The present HPLC-DAD-ESIMS analytical method was successfully used to separate and determinate various types of compounds in the complex mixture. Moreover, it is notable that several identified compounds in this fraction have been reported beneficial effect in atherosclerosis and related ailments. Ferulic acid, ${ }^{37,38)}$ auraptene ${ }^{39)}$ saikosaponins ${ }^{40)}$ were reported to be anti-inflammatory. Flavonoids, such as naringenin, hersperetin and related derivatives were found hypolipidemia ${ }^{41,42)}$ and phytoestrogen $^{43)}$ activity. Several phthalides were reported inhibitory effect on proliferation of aorta smooth muscle cells. ${ }^{44)}$ Taken together, we suggested that multiple bioactive components were responsible for the therapeutical effect of Xue-Fu-Zhu-Yu-Tang. Our finding may help to clarify the re- medial mechanisms and develop a reliable quality control method for the age-old TCM formula. Moreover, we also showed that collective application of bioassays and HPLCDAD-ESI-MS analysis was an approach to rapidly characterize bioactive constituents in TCM formulae.

Acknowledgements This study was supported by Chinese National Basic Research Priorities Program-973 (No. G1999054405) and Key Research Program of National Science Foundation of China (No. 90209005). The author would like to thank Beijing Xiyuan Hospital for the animal experiments and Mr. Yecheng Xiao for ${ }^{1} \mathrm{H}-\mathrm{NMR}$ analysis and his valuable advise.

\section{References}

1) New D. C., Miller-Martini D. M., Wong Y. H., Phytother. Res., 17, $439-448$ (2003).

2) Yuan R., Lin Y., Pharmacol. Ther., 86, 191-198 (2000).

3) Gong X., Sucher N. J., Trends Pharmacol. Sci., 9, 191-196 (1999).

4) Strege M. A., J. Chromatogr. B, Biomed. Appl., 725, 67-78 (1999).

5) Julian R. K., Higgs R. E., Gygi J. D., Hilton M. D., Anal. Chem., 70, 3249-3254 (1998).

6) Cremin P. A., Zeng L., Anal. Chem., 74, 5492-5500 (2002).

7) Lee M. S., Kerns E. H., Mass Spectrom. Rev., 18, 187-279 (1999).

8) He X. G., Lin L. Z., Lian L. Z., J. Chromatogr. A, 755, 127-132 (1996).

9) He X. G., Lian L. Z., Lin L. Z., Bernart M. B., J. Chromatogr. A, 791, 127-134 (1997).

10) Li S. L., Chan S., Lin G., Ling L., Yan R., Chung H. S., Tam Y. K., Planta Med., 69, 445-451 (2003).

11) Borbalan A. M. A., Zorro L., Guillen D. A., Barroso C. G., J. Chromatogr. A, 1012, 31-38 (2003).

12) Cai Z. W., Lee F., Wang X. R., Yu W. J., J. Mass Spectrom., 37, 1013-1024 (2002). 
13) Li Y. M., Chen K. J., Shi Z. X. Zhang T. Z., Zhongguo Zhong Xi Yi Jie He Za Zhi, 18, 71-73 (1998).

14) Muegge I., Med. Res. Rev., 23, 302-321 (2003).

15) Kobayashi M., Fujita M., Mitsuhashi H., Chem. Pharm. Bull., 35, 1427-1433 (1987).

16) Redl K., Breu W., Davis B., Bauer R., Planta Med., 60, 58-62 (1994).

17) Kobayashi M., Fujita M., Mitsuhashi H., Chem. Pharm. Bull., 32, $3770-3773$ (1984).

18) Mitsuhashi H., Nagai U., Tetrahedron, 19, 1277-1283 (1963).

19) Allen E. H., Thomas C. A., Phytochemistry, 10, 1579-1582 (1971).

20) Nakada H., Kobayashi A., Yamashita K., Agric. Biol. Chem., 41, 1761-1765 (1977).

21) Bauer R., Redl K., Davis B., Phytochemistry, 31, 2035-2037 (1992).

22) Chatterjee A., Dutta C. P., Bhattacharyya S., Audier H. E., Das B. C., Tetrahedron Lett., 8, 471- 473 (1967).

23) Yamada Y., Nakatani N., Fuwa H., Agric. Biol. Chem., 51, 11051110 (1987).

24) Vogel H. G., Vogel W. H., "Drug Discovery and Evaluation: Pharmacology Assays," Springer-Verlag, Berlin, 1997.

$25) \quad$ Illingworth D. R., Clin. Invest. Med., 13, 211-218 (1990).

26) Gryglewski R. J., Ann. Med., 27, 421-427 (1995).

27) Ross R., N. Engl. J. Med., 14, 115-121 (1999).

28) Kamiya A., Esaki S., Konishi F., Agric. Biol. Chem., 43, 1529-1539 (1979).

29) Wang P. S., Gao X. L., Wang Y. X., Fukuyama Y., Miura I., Sugawara M., Phytochemistry, 23, 2033-2038 (1984).

30) Kaouadji M., Pachterf F. D., Pouget C., Chulia A. J., J. Nat. Prod., 49, $872-877$ (1986)
31) Mitsuhashi H., Muramatsu T., Tetrahedron, 20, 1971-1982 (1964).

32) Kaneda M. Iitaka Y., Shibata S., Tetrahedron, 28, 4309- 4317 (1972).

33) Ruth J., Ferdinand B., Schöneweiss S., Phytochemistry, 18, 829-837 (1979).

34) Ishii H., Nakamura M., Seo S., Tori K., Tozyo T., Yoshimura Y., Chem. Pharm. Bull., 28, 2367-2383 (1980)

35) Liang X. T., "National Natural Science Foundation of China, Fundamental Research on Commonly Used TCM Herbs," Vol. I., Science Press, Beijing, 2003.

36) Masuda T., Muroya Y., Nakatani N., Phytochemisty, 31, 1363-1366 (1992).

37) Maggi-Capeyron M. F., Ceballos P., Cristol J. P., Delbosc S., Le Doucen C., Pons M., Leger C. L., Descomps B., J. Agric. Food Chem., 49, 5646-5652 (2001).

38) Sakai S., Ochiai H., Nakajima K., Terasawa K., Cytokine, 9, 131-137 (1997).

39) Murakami A., Nakamura Y., Tanaka T., Kawabata K., Takahashi D., Koshimizu K., Ohigashi H., Carcinogenesis, 21, 1843-1850 (2000).

40) Ushio Y., Oda Y., Abe H., Int. J. Immunopharmacol., 13, 501-508 (1991).

41) Seo H. J., Jeong K. S., Lee M. K., Park Y. B., Jung U. J., Kim H. J., Choi M. S., Life Sci., 73, 933-946 (2003).

42) Kima H. K., Jeongb T. S., Leea M. K., Parkc Y. B., Choia M. S., Clin. Chim. Acta, 327, 129-137 (2003).

43) Zierau O., Gester S., Schwab P., Metz P., Kolba S., Wulf M., Vollmer G., Planta Med., 68, 449-451 (2002).

44) Kobayashi S., Mimura Y., Naitoh T., Kimura I., Kimura M., Jpn. J. Pharmacol., 63, 353-359 (1993). 